\title{
The Unitarity Problem and the Zero-Mass Limit for a Model of Massive Yang-Mills Theory.
}

G. CurCI

Scuola Normale Superiore - Pisa, Italia

R. FERRari

Max-Planok-Institut für Physik und Astrophysik - München, Fed. Rep. of Germany (Nuovo Oimento, 35 A, 1 (1976))

In this paper we discussed a model of non-Abelian gauge theory where the vector meson has a finite mass. The model gives an action invariant under Becchi, Ronet and Stora transformations. However, physical unitarity is violated. We claimed that this property is restored in the limit of zero mass; in this way the model could give a nice way of regularizing the infra-rod divergences.

Our proof is unfortunately incorrect. In particular at the end of sect. 4 we have neglected the fact that the spin-one part of the propagator can have a residuum different from the one of the spin-zero part. Thus eq. (40) is incorrect. Moreover eq. (39) is wrong for $\alpha \neq 1$. Therefore, we do not prove that physical unitarity is recovered in the zeromass limit. We hope to further discuss this problem in a future publication (").

The following points of the paper are incorrect:

Summary: ... We show how these properties are restored in the limit of zero mass.

Pag. 2 line 8: ... Then we show how physical unitarity is restored in the limit of zero mass.

Pag. 9: From line 12 to the end of sect. 4.

(*) In order to recover physical unitarity, a better definition of the physical space is probably needed. In this respect see the discussion of gauge invariance of transition probabilities by G. CoRCI and E. D'FinIo: On gauge invariance and perturbative calcula. tions in quantum chronodynamics, CERN, preprint (1978). 